\title{
Induced Flow and Optical Emission Generated by a Pulsed 13.56 MHz-5 kHz Plasma Actuator
}

\author{
James Dedrick, Seong-kyun Im, Mark Cappelli, Rod W. Boswell, and Christine Charles
}

\begin{abstract}
A dual-frequency driven plasma actuator is studied with respect to the influence of changing the position of radio frequency $13.56 \mathrm{MHz}$ pulses within the phase of a low-frequency (LF) $5 \mathrm{kHz}$ sinusoidal voltage. Fast imaging and 2-D particle image velocimetry are used to study the optical emission from the discharge and the generation of an induced flow, which can be useful for aerodynamic flow control, in ambient air. The results show that the propagation of the discharge away from the powered electrode and the velocity of the induced flow are maximized when the pulses are positioned at the peaks of the LF waveform. In contrast, the most intense optical emission is found to occur when the pulses are positioned close to the zero crossings for positive $\mathrm{LF}$ waveform voltages. The reasons behind these observations are discussed.
\end{abstract}

Index Terms-Actuators, flow control, optical imaging, plasma applications, surface discharges.

\section{INTRODUCTION}

$\mathbf{I}$ $\mathrm{N}$ the context of active flow control research, the application of ionized-gas discharges to the modification of boundary layers has received significant attention in recent years [1]. One promising design is the asymmetric surface dielectric barrier discharge (ASDBD), in which an ac high-voltage waveform of hertz-kilohertz is applied between two electrodes that are asymmetrically affixed about a layer of dielectric material. ASDBDs are of simple construction, lightweight, have no moving parts, and are capable of rapid response times. Since they generate a non-thermal discharge [2], flow actuation is achievable with relatively modest power requirements [3]-[5].

Over the last decade, the performance of ASDBD plasma actuators has been studied with respect to applications including the control of boundary layer separation [6], modification of supersonic flows [7], vortex generation [8], and noise attenuation [9].

A current challenge in the development of plasma actuators is their limited authority in high Reynolds number flows,

Manuscript received April 30, 2013; revised July 23, 2013 and August 8, 2013; accepted August 9, 2013. Date of publication October 16, 2013; date of current version December 9, 2013. This work was supported in part by the SP3 Laboratory and in part by The Australian National University.

J. Dedrick, R. W. Boswell, and C. Charles are with the Space Plasma, Power and Propulsion Laboratory, Research School of Physics and Engineering, The Australian National University, Canberra ACT 0200, Australia (e-mail: james.dedrick@anu.edu.au; rod.boswell@anu.edu.au; christine.charles@anu.edu.au).

S. Im and M. Cappelli are with the Mechanical Engineering Department, Stanford University, Stanford, CA 94305-3032 USA (e-mail: sim3@stanford.edu; cap@stanford.edu).

Color versions of one or more of the figures in this paper are available online at http://ieeexplore.iee.org.

Digital Object Identifier 10.1109/TPS.2013.2279879 i.e., $\operatorname{Re}>10^{5}$ [4]. This has motivated significant effort in the optimization of the actuator design [10] and recent studies have investigated the effect of using non-sinusoidal voltage waveforms [11], [12], the modification of the electrode shape [13], the thickness of the dielectric layer [14], and the use of a three-electrode configuration to extend the surface area over which actuation is achieved [15]. Significant efforts have also been undertaken to understand the mechanism behind the generation of thrust. Recent work has suggested that actuation may be caused by a momentum transfer between discharge ions, which drift due to the electric field created between the electrodes, and neutrals in the freestream [16], [17].

To increase flow-control authority, it may be of benefit to increase the density of ions in the discharge and hence maximize momentum transfer to neutrals. Radio frequency (RF) discharges, for which the voltage oscillates at several megahertz, are understood to be effective in increasing the density of atmospheric-pressure plasmas, as investigated in [18].

In this paper, pulses of RF voltage are combined with a low-frequency (LF) sinusoidal voltage waveform [19]. The RF waveform is used to increase the production of ions above that generated by an LF waveform separately, but oscillates too rapidly for significant drift to occur within the pulse. Subsequent to the end of the pulse, the ions may be accelerated in the LF field and transfer momentum to neutrals. The effect of changing the position of the RF pulse within the LF waveform period is studied with respect to the generation of an induced flow and the optical emission, because these are indicators of the degree of actuation and the spatial distribution of ionization and power deposition, respectively.

\section{EXPERIMEntal Configuration}

The actuator is constructed with copper-tape electrodes (35 $\mu \mathrm{m}$ thick, powered electrode $10 \mathrm{~mm}$ wide, and grounded electrode $19 \mathrm{~mm}$ wide) that are separated by a dielectric layer of Kapton tape ( $80 \mu \mathrm{m}$ thick). As shown in Fig. 1, the electrodes are offset in the $x$-direction such that the discharge propagates out over the surface of the dielectric layer.

The spanwise length of the actuator is $20 \mathrm{~cm}$ and is directed along the $z$-axis in Fig. 1. The actuator is mounted onto a rigid dielectric plate and installed inside the test section of a low-speed wind tunnel to ensure stagnant flow conditions with negligible imposed flow. All experiments are undertaken at atmospheric pressure using the ambient air of the laboratory.

As shown in Fig. 2, a dual-frequency voltage waveform, which comprises $5 \mu$ s pulses of RF $13.56 \mathrm{MHz}$ voltage at 


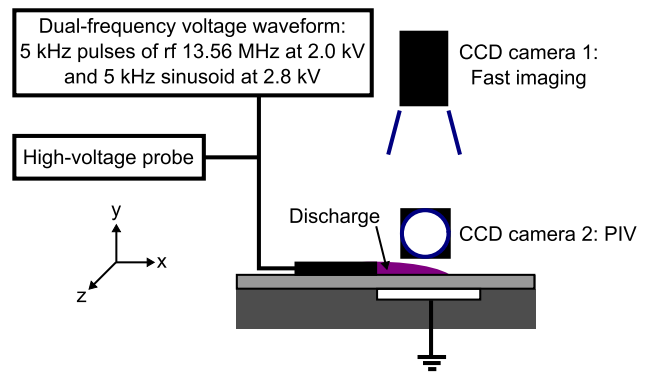

Fig. 1. Schematic of the experiment apparatus showing the surface discharge plasma actuator and the orientation of the cameras for particle image velocimetry (PIV) and fast imaging. The powered electrode, grounded electrode, dielectric layer, and mounting plate are shown in black, white, light gray, and dark gray, respectively. Fast imaging and PIV are undertaken in the $x z$ and $x y$ planes, respectively.

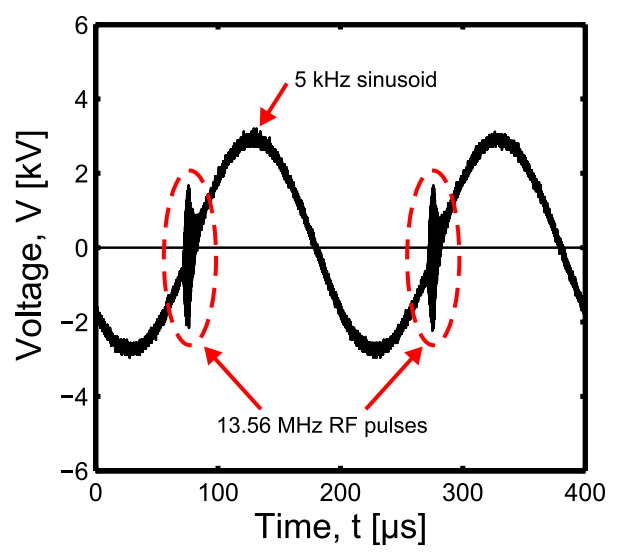

Fig. 2. Variation in the dual-frequency voltage applied to the actuator as measured at the powered electrode with the high-voltage probe. The pulses of $13.56 \mathrm{MHz} \mathrm{RF}$ voltage that are combined with the sinusoidal $5 \mathrm{kHz} \mathrm{LF}$ voltage waveform at $t=75$ and $t=275 \mu$ s (enclosed with dashed lines) are shown as envelopes to aid visualization.

$2.0 \mathrm{kV}$ and $5 \mathrm{kHz}$ (period $200 \mu \mathrm{s}$ ) together with a sinusoidal, $5 \mathrm{kHz} \mathrm{LF}$ waveform at $2.8 \mathrm{kV}$, is applied at the powered electrode and monitored using a high-voltage probe (dc to $75 \mathrm{MHz}$ ). In this configuration, there is one pulse of RF voltage per period of the LF waveform. The amplitude and frequency of both waveforms is held constant while the position of the RF pulse within the phase of the LF waveform period is varied, e.g., Fig. 2 shows an RF pulse position of zero phase.

To study the effect of changing the RF pulse position, two optical diagnostic tools are employed. A charge coupled device camera (CCD: LaVision Imager Intense, $1024 \times 1374$ pixels, $50 \mathrm{~mm}$ lens, 12 bit, spatial resolution 129 pixels $/ \mathrm{mm}$ ), which is oriented parallel to the plane of the dielectric surface, as shown by CCD camera 1 in Fig. 1, is used to detect the optical emission of the discharge. The exposure time is $1 \mathrm{~ms}$ (all the images are single shots and no accumulations are used) and this corresponds to five oscillations of the LF waveform (each $200 \mu \mathrm{s})$ at $5 \mathrm{kHz}$. Because the parameters of primary interest are the intensity of the optical emission and the propagation length of the discharge away from the powered electrode ( $x$-direction) the intensity is averaged over the actuator length (z-direction).
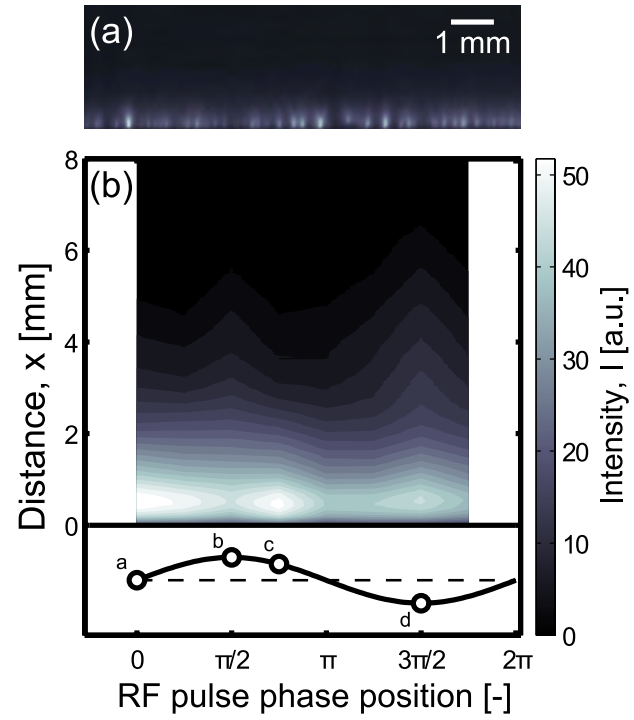

Fig. 3. (a) Image of the discharge for an RF pulse position of zero phase (see Fig. 2). The edge of the powered electrode corresponds to the bottom edge of the image and the intensity is normalized with respect to its own maximum for clarity. (b) Variation in the intensity of the optical emission from the discharge, averaged over the actuator length ( $z$-axis), with respect to the position of the RF pulse within the phase of the LF waveform. The edge of the powered electrode is located at a distance of $x=0 \mathrm{~mm}$ and the relative phase of the LF waveform is shown at the bottom. One period of the $5 \mathrm{kHz}$ LF waveform corresponds to $200 \mu \mathrm{s}$, as shown in Fig. 2. The phase positions for which velocity fields are shown in Fig. 4 are marked by open circles (a)-(d).

2-D particle image velocimetry (PIV) is used to measure the motion of the ambient air surrounding the actuator and hence gauge the effect of the RF pulse position on the generation of an induced flow. The PIV setup employs an Nd:YAG laser (New wave Gemini PIV, wavelength $532 \mathrm{~nm}$, repetition rate $3 \mathrm{~Hz}$ ), which is used to generate a thin (about $1 \mathrm{~mm}$ thick) laser sheet and this is directed toward the actuator in the $x y$ plane midway along the actuator length. A second CCD camera (LaVision Imager Intense, $1024 \times 1280$ pixels, $50 \mathrm{~mm}$ lens, 12 bit), which is numbered 2 in Fig. 1, is fitted with a $532 \mathrm{~nm}$ bandpass filter (full width at half maximum $10 \mathrm{~nm}$ ) and is used to detect the laser light scattered from submicrometer aluminum oxide particles. These are blown into the test section using compressed air (the wind tunnel fan is only used between tests to flush the test section) and are assumed to follow, or trace, the motion of the air surrounding the actuator. The $45 \mathrm{~mm} \times 15 \mathrm{~mm}$ field-of-view for PIV is located in the $x y$ plane and the absence of any background motion within this region is confirmed when the discharge is inactive.

Velocity fields are generated using the average result of 300 PIV image pairs. The delay between successive images in each pair is $700 \mu \mathrm{s}$. DaVis 7 software is employed for the cross-correlation routine, which consists of a multipass method with a $50 \%$ overlap using windows of dimension $64 \times 64$ pixels followed by $32 \times 32$ pixels. The resultant spatial resolution of the velocity vectors and the estimated uncertainty are 0.75 vectors $/ \mathrm{mm}$ and $1.15 \%$, respectively.

\section{RESUlTS}

The span-averaged optical emission, as detected from the discharge by CCD camera 1, is shown in Fig. 3 for a series of 

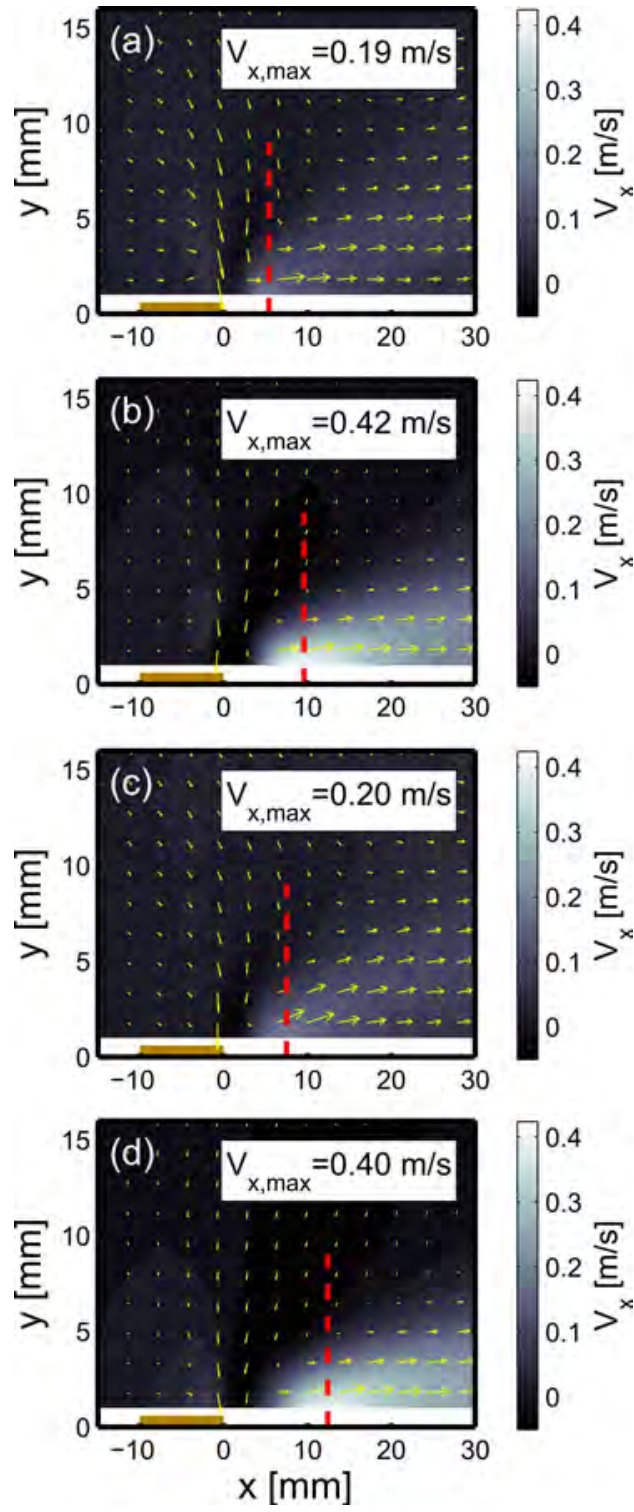

Fig. 4. Spatial variation in the induced flow generated by the actuator when the RF pulse is positioned at (a) zero phase, (b) $\pi / 2$, (c) $3 \pi / 4$, and (d) $3 \pi / 2$ as marked by the open circles in Fig. 3. The powered electrode is shown by the solid line for $-10 \geq x \leq 0 \mathrm{~mm}$ (thickness not to scale) and the surface of the dielectric is located at $y=0 \mathrm{~mm}$. The component of velocity in the $x$-direction $V_{x}$ and the total velocity are shown by the colors and arrows, respectively, and the maximum velocity $V_{x, \max }$ is noted. The distances from the powered electrode at which $V_{x, \max }$ is measured are shown by the dashed lines.

RF pulse positions throughout the period of the LF waveform. It is observed that the intensity of the optical emission and the length to which the discharge propagates in the $x$-direction, i.e., away from the powered electrode edge over the dielectric layer, are dependent upon where the RF pulse is positioned.

The most intense optical emission is observed when the pulse is positioned where the rate of change of the LF waveform is relatively large and occurs close to the zero crossings during the positive half-period only, i.e., at (a) and (c). In contrast, the propagation length of the discharge is maximized when the RF pulse is positioned at the maxima and minima of the LF waveform at (b) and (d), respectively. This corresponds to phase positions of $\pi / 2$ (LF waveform maximum) and $3 \pi / 2$ (LF waveform minimum) and is in agreement with previous findings for an ASDBD presented in [20], for which the propagation length was observed to increase (linearly) for increasing voltage amplitudes.

The velocity fields generated in the region near to the actuator for the RF pulse positions (a)-(d) are shown in Fig. 4. The velocity of the induced flow tangential to the dielectric surface $V_{x}$ is maximized when the RF pulse is positioned at the peaks of the LF waveform, as shown by (b) and (d) for the positions $\pi / 2$ and $3 \pi / 2$, respectively. This corresponds to the positions for which the discharge reaches its maximum extent of propagation (see Fig. 3) and is consistent with the findings in [21].

It is observed that the pulse positions for which the optical emission is most intense, i.e., points (a) and (c) in Fig. 3, the maximum velocity of the induced flow $V_{x, \max }$ is only $0.19 \mathrm{~m} / \mathrm{s}$ and $0.20 \mathrm{~m} / \mathrm{s}$, or $45 \%$ and $48 \%$ of the maximum of $0.42 \mathrm{~m} / \mathrm{s}$ at point (b), respectively. This suggests that although the degree of ionization may be greatest for pulse positions (a) and (c), the acceleration of ions is enhanced for positions (b) and (d), i.e., when the electric field due to the LF waveform is maximized at the end of each RF pulse. This is consistent with the understanding that for the increased generation of an induced flow, both the ion density and the ion drift velocity should be maximized. At points (a) and (c), even though a higher ion density may be created through enhanced ionization, at the end of each RF pulse the electric field due to the LF waveform is relatively low compared with that at points (b) and (d).

As shown by the dashed lines in Fig. 4, the distance from the edge of the powered electrode at which the maximum induced flow velocity $V_{x, \max }$ occurs also depends upon the position of the pulse. For pulse positions at the maxima and minima of the LF waveform, i.e., points (b) and (d), the maximum flow velocity is measured $9.6 \mathrm{~mm}$ and $12.4 \mathrm{~mm}$ away from the powered electrode, respectively. In contrast, during the rising and falling intervals, i.e., points (a) and (c), the distance from the powered electrode at which actuation is maximized is smaller at $5.4 \mathrm{~mm}$ and $7.5 \mathrm{~mm}$, respectively. As shown in Fig. 3, increasing LF voltage at the end of the RF pulse is correlated with an increasing propagation distance of the discharge and this may result in the increased separation of the flow-control region from the powered electrode.

\section{CONCLUSION}

Fast imaging and particle image velocimetry have been used to study a pulsed $13.56 \mathrm{MHz}-5 \mathrm{kHz}$ plasma actuator regarding the effect of the pulse position within the phase of the $5 \mathrm{kHz}$ voltage waveform. The results show that the velocity of the induced flow, and the distance from the powered electrode at which the maximum velocity is observed, is largest when the RF pulses are positioned at the maxima and minima of the lower frequency waveform. This is distinct from the optical emission, which is observed to be most intense when the pulses are positioned close to the zero crossings of the lower frequency waveform during the positive halfperiod. The observation that the maximum discharge extension and maximum flow velocity in the direction tangential to the dielectric occur when the peak driving voltage is maximized is in agreement with the previous studies for LF-driven ASDBDs. 


\section{ACKNOWLEDGMENT}

The authors would like to thank P. Alexander, S. Holgate, and D. Tempra for their technical assistance.

\section{REFERENCES}

[1] L. N. Cattafesta III and M. Sheplak, "Actuators for active flow control," Annu. Rev. Fluid Mech., vol. 43, pp. 247-272, Jan. 2011.

[2] A. Fridman, A. Chirokov, and A. Gutsol, "Non-thermal atmospheric pressure discharges," J. Phys. D, Appl. Phys., vol. 38, no. 2, pp. R1-R24, 2005.

[3] J. Reece Roth, "Aerodynamic flow acceleration using paraelectric and peristaltic electrohydrodynamic effects of a one atmosphere uniform glow discharge plasmaa," Phys. Plasmas, vol. 10, no. 5, pp. 2117-2126, 2003.

[4] E. Moreau, "Airflow control by non-thermal plasma actuators," J. Phys. D, Appl. Phys., vol. 40, no. 3, pp. 605-636, 2007.

[5] T. C. Corke, C. L. Enloe, and S. P. Wilkinson, "Dielectric barrier discharge plasma actuators for flow control," Annu. Rev. Fluid Mech., vol. 42, pp. 505-529, 2010.

[6] J. Little, M. Nishihara, I. Adamovich, and M. Samimy, "High-lift airfoil trailing edge separation control using a single dielectric barrier discharge plasma actuator," Experim. Fluids, vol. 48, no. 3, pp. 521-537, 2010.

[7] S. Im, H. Do, and M. A. Cappelli, "Dielectric barrier discharge control of a turbulent boundary layer in a supersonic flow," Appl. Phys. Lett., vol. 97, no. 4, pp. 041503-1-041503-3, 2010.

[8] T. N. Jukes and K. S. Choi, "Dielectric-barrier-discharge vortex generators: Characterisation and optimisation for flow separation control," Experim. Fluids, vol. 52, no. 2, pp. 329-345, 2012.

[9] X. Huang, S. Chan, and X. Zhang, "Atmospheric plasma actuators for aeroacoustic applications," IEEE Trans. Plasma Sci., vol. 35, no. 3, pp. 693-695, Jun. 2007.

[10] M. Forte, J. Jolibois, J. Pons, E. Moreau, G. Touchard, and M. Cazalens, "Optimization of a dielectric barrier discharge actuator by stationary and non-stationary measurements of the induced flow velocity: Application to airflow control," Experim. Fluids, vol. 43, no. 6, pp. 917-928, 2007.

[11] D. F. Opaits, M. N. Shneider, R. B. Miles, A. V. Likhanskii, and S. O. Macheret, "Surface charge in dielectric barrier discharge plasma actuators," Phys. Plasmas, vol. 15, no. 7, pp. 073505-1-073505-5, 2008.
[12] N. Benard and E. Moreau, "Role of the electric waveform supplying a dielectric barrier discharge plasma actuator," Appl. Phys. Lett., vol. 100, no. 19, pp. 193503-1-193503-5, 2012.

[13] A. R. Hoskinson and N. Hershkowitz, "Differences between dielectric barrier discharge plasma actuators with cylindrical and rectangular exposed electrodes," J. Phys. D, Appl. Phys., vol. 43, no. 6, pp. 065205-1-065205-8, 2010.

[14] F. O. Thomas, T. C. Corke, M. Iqbal, K. Kozlov, and D. Schatzman, "Optimization of dielectric barrier discharge plasma actuators for active aerodynamic flow control," AIAA J., vol. 47, no. 9, pp. 2169-2178, 2009.

[15] E. Moreau, R. Sosa, and G. Artana, "Electric wind produced by surface plasma actuators: A new dielectric barrier discharge based on a threeelectrode geometry," J. Phys. D, Appl. Phys., vol. 41, no. 11, pp. 115204 1-115204-12, 2008.

[16] J. P. Boeuf, Y. Lagmich, Th. Unfer, Th. Callegari, and L. C. Pitchford, "Electrohydrodynamic force in dielectric barrier discharge plasma actuators," J. Phys. D, Appl. Phys., vol. 40, no. 3, pp. 652-662, 2007.

[17] J. P. Boeuf, Y. Lagmich, and L. C. Pitchford, "Contribution of positive and negative ions to the electrohydrodynamic force in a dielectric barrier discharge plasma actuator operating in air," J. Appl. Phys., vol. 106, no. 2, pp. 023115-1-023115-9, 2009.

[18] D. B. Kim, J. K. Rhee, B. Gweon, S. Y. Moon, and W. Choe, "Comparative study of atmospheric pressure low and radio frequency microjet plasmas produced in a single electrode configuration," Appl. Phys. Lett., vol. 91, no. 15, pp. 151502-1-151502-3, 2007.

[19] J. Dedrick, S. Im, M. A. Cappelli, R. W. Boswell, and C. Charles, "Surface discharge plasma actuator driven by a pulsed $13.56 \mathrm{MHz}-5 \mathrm{kHz}$ voltage waveform," J. Phys. D, Appl. Phys., vol. 46, no. 40, pp. 405201-1-405201-8, 2013.

[20] K. Allegraud, O. Guaitella, and A. Rousseau, "Spatio-temporal breakdown in surface DBDs: Evidence of collective effect," J. Phys. D, Appl. Phys., vol. 40, no. 24, pp. 7698-7706, 2007.

[21] R. Durscher and S. Roy, "Evaluation of thrust measurement techniques for dielectric barrier discharge actuators," Experim. Fluids, vol. 53, no. 4, pp. 1165-1176, 2012

Authors' photographs and biographies not available at the time of publication. 